\title{
THE SPECTRUM OF VECTOR BUNDLE FLOWS WITH INVARIANT SUBBUNDLES
}

\author{
R. C. SWANSON ${ }^{1}$
}

\begin{abstract}
A vector bundle flow $\left(\Phi^{t}, \phi^{t}\right)$ on the vector bundle $E$ over a compact metric space $M$ induces a one-parameter group $\left\{\Phi_{t}^{\#}\right\}$ of bounded operators acting on the continuous sections of $E$, with infinitesimal generator $L$. An example is given by the tangent flow $\left(T \phi^{t}, \phi^{t}\right)$, if $\phi^{t}$ is a flow on a smooth manifold. In this article, the spectrum of the generator $L$ is used to study the exponential growth rates of bundle trajectories in the neighborhood of a fixed invariant subbundle, e.g. the tangent bundle of a submanifold of $\boldsymbol{M}$. Auxiliary normal and tangential spectra are introduced, and their relationship and fine structure are explored.
\end{abstract}

Introduction. The aim of this paper is to link linear dynamics in vector bundles to the spectra of certain Banach space operators associated with a linear vector bundle flow. Of particular interest is the behavior of such systems at a fixed invariant subbundle, e.g. the tangent bundle of an invariant submanifold, discussed below. What sets this article apart from related work of, say, Sacker and Sell [8], [9] is a faithfulness to the operator-theoretic point of view, which allows one to sieve out the "easy" functional analysis from the harder dynamics. The payoff comes if difficult results become natural and easy-to-state facts about operators, or, conversely, statements about the spectrum lead to new dynamical considerations. Specific examples of such dividends are given in [1], [5] as well as in this paper.

In $\$ 1$ we introduce the pertinent notation and terminology (see also [1]); while in $\S 2$, we isolate results from abstract operator theory which are then applied, in $\$ 3$, to vector bundle flows with a fixed invariant subbundle. As in [9], we identify the tangential spectrum along the subbundle, $\sigma\left(L_{F}\right)$, and the normal spectrum $\sigma(\bar{L})$; but as operator spectra, yielding the inclusion $\sigma(L) \subset \sigma\left(L_{F}\right) \cup \sigma(\bar{L})$ (Theorem 3.2), which implies Theorem 1 of [9]. Next, sufficient conditions are given for the crucial equality $\sigma(L)=\sigma\left(L_{F}\right) \cup \sigma(\bar{L})$, which means, roughly, that the growth rates of the bundle trajectories can be determined from the normal and tangential dynamics. We describe a flow example showing that the equality can fail. Theorem 3.5 generalizes the result in [9] asserting that equality always holds if the base flow is chain recurrent. Finally, we discuss the existence of complementary invariant subbundles in terms of operator theory.

1. The spectrum of a vector bundle flow. Let $M$ denote a compact metric space which admits a one-parameter group of homeomorphisms $\left\{\phi^{t}\right\}_{t \in \mathbf{R}}$. Suppose $E$ is a

Received by the editors June 30, 1980.

1980 Mathematics Subject Classification. Primary 58F15, Secondary 58F 19.

Key words and phrases. Vector bundle flows, infinitesimal generator of a semigroup, operator theory.

${ }^{1}$ Research partially supported by the University of Missouri Graduate Research Council. (C) 1981 American Mathematical Society 0002-9939/81/0000-0432/\$02.25 
continuous complex vector bundle over $M$ with projection map $\pi: E \rightarrow M$. We assume further that $E$ is equipped with a one-parameter group of (linear) vector bundle automorphisms $\Phi^{t}: E \rightarrow E$ which covers the base flow: $\pi \Phi^{t}=\phi^{t} \pi$.

When $M$ is a smooth manifold, an important example is provided by the tangent flow. In that case, $\left\{\phi^{t}\right\}$ is generated by a $C^{1}$ vector field $X$ on $M$, while the vector bundle flow $\left\{\Phi^{t}\right\}$ corresponds to $T \phi^{t}$ acting on the tangent bundle $T M$.

We may suppose $E$ admits a continuous Finsler structure with fiber norm, $|\cdot|=|\cdot|_{x}$. By compactness, the space $\Gamma(E)$ of continuous cross sections of $E$ is independent of the choice of Finsler.

Define a continuous group $\left\{\Phi_{t}^{\sharp}\right\}, t$ in $\mathbf{R}$, of bounded operators on $\Gamma(E)$ by setting $\Phi_{t}^{\sharp} \eta=\Phi^{-t} \eta \phi^{t}$, if $\eta$ is in $\Gamma(E)$. We impose the mild restriction that $\Phi_{t}^{\#}$ is a $C_{0}$-semigroup [4], which always holds for smooth vector bundle flows. The infinitesimal generator,

$$
L \eta=\left.\frac{d}{d t} \Phi_{t}^{\sharp} \eta\right|_{t=0},
$$

is called the Lie derivative along $\Phi^{t}$. For tangent flows, $L$ is the usual Lie derivative along the vector field.

A good deal is known about the operator $L$, which is a closed first order differential operator with dense domain. Let $\sigma(L)$ denote the usual operator spectrum of $L$. Sacker and Sell [8] have introduced a "spectrum" $\Sigma$ of real numbers-roughly, the exponential growth rates of bundle trajectories-which we shall refer to as the Sacker-Sell spectrum of the bundle flow.

Up to imaginary translation, $\Sigma$ behaves like a true operator spectrum:

Proposition 1.1 [2, TheOREM 3.2]. $\Sigma=\operatorname{Re}[\sigma(L)]$.

Let $\mathbb{Q}(S)$ denote the union of these circles about the origin which meet the set $S$ of complex numbers. The following exponentiation result is proved in [2].

THEOREM 1.2. Let $\Phi_{t}^{\sharp}$ denote the group of bounded operators on $\Gamma(E)$ generated by $L$. Then the following relations obtain:

$$
e^{t \sigma(L)} \subset \sigma\left(\Phi_{t}^{\sharp}\right) \subset \mathbb{Q}\left[e^{t \sigma(L)}\right] .
$$

REMARKs. The equality $e^{t \sigma(L)}=\sigma\left(\Phi_{t}^{\sharp}\right)$ holds quite generally. If the nonperiodic points of $\Phi^{t}$ are dense, for a smooth flow, then equality holds. Equality also holds for nonsingular tangent flows [1].

2. Linear operators with invariant subspaces. In this section $A$ denotes any linear operator, possibly unbounded, having closed graph and dense domain $D(A)$, which acts in the complex Banach space $X$. For example, the Lie differentiation operator $L$ has these properties.

The spectrum $\sigma(A)$ consists of all complex numbers $\lambda$ such that $A-\lambda$ fails to admit a bounded inverse defined on all of $X$. Let $Y$ be a fixed closed subspace of $X$ such that $A(D(A) \cap Y) \subset Y$, and let $A_{Y}=A \mid D(A) \cap Y$. We also have the quotient operator $\bar{A}$ defined in the Banach space $X / Y$ with domain $\pi(D(A))$, where $\pi$ : $X \rightarrow X / Y$ is the usual projection. 
We now list some results for closed operators which are well known for bounded operators with an invariant subspace. Property (i) is given in [5, p. 15], while (ii) is in [3, p. 24]. Statement (iii) is immediate from (ii). The proofs are similar to the bounded case.

Proposition 2.1. For an operator $A$ in $X$ with closed graph and dense domain, the spectra $\sigma(A), \sigma\left(A_{Y}\right)$ and $\sigma(\bar{A})$ are such that

(i) $\sigma(A) \subset \sigma\left(A_{Y}\right) \cup \sigma(\bar{A})$.

(ii) $\sigma\left(A_{Y}\right) \backslash \sigma(A)=\sigma(\bar{A}) \backslash \sigma(A)$.

(iii) If $\sigma\left(A_{Y}\right) \cap \sigma(\bar{A}) \subset \sigma(A)$, then

$$
\sigma(A)=\sigma\left(A_{Y}\right) \cup \sigma(\bar{A}) .
$$

Define, as in [3, p. 8], the approximate point spectrum, written $\sigma_{\mathrm{ap}}(A)$, to consist of complex numbers $\lambda$ such that there is a sequence $\left\{x_{n}\right\}$ in $X$ with $\left\|x_{n}\right\|=1$ and $\lim _{n \rightarrow \infty}\left\|(A-\lambda) x_{n}\right\|=0$.

The pertinent properties of $\sigma_{\mathrm{ap}}(A)$ are summarized below. We shall continue to assume the hypotheses of Proposition 2.1.

Proposition 2.2. If $\sigma_{\mathrm{ap}}(A)$ is the approximate point spectrum, then

(i) The boundary of $\sigma(A)$ is contained in $\sigma_{\mathrm{ap}}(A)$.

(ii) $\sigma_{\text {ap }}\left(A_{Y}\right) \subset \sigma_{\text {ap }}(A)$.

(iii) If $\sigma\left(A_{Y}\right)=\sigma_{\mathrm{ap}}\left(A_{Y}\right)$, then $\sigma(A)=\sigma\left(A_{Y}\right) \cup \sigma(\bar{A})$.

Proof. Statement (i) is well known, and the proof in [3, p. 9] is valid for unbounded operators. Property (ii) is obvious. Together, Proposition 2.1(ii) and (ii) above, imply the fact (iii).

The last result of this section, dealing with the existence of invariant complements, is an easy consequence of the Riesz decomposition theorem [7, p. 421] and Proposition 2.1(i).

Proposition 2.3. Let $A$ be a bounded operator on $X$ such that $\sigma\left(A_{Y}\right) \cap \sigma(\bar{A})=\varnothing$. Then there exists a unique closed subspace $Z$ in $X$ such that

(i) $X=Y \oplus Z, A Z \subset Z$, and $\sigma(\bar{A})=\sigma\left(A_{Z}\right)$.

(ii) $\sigma(A)=\sigma\left(A_{Y}\right) \cup \sigma(\bar{A})$.

3. The normal and tangential spectra. As in $\S 1$, suppose that a continuous vector bundle flow $\left(\Phi^{t}, \phi^{t}\right)$ acts on the vector bundle $(E, M)$ and gives rise to a $C_{0}$-semigroup of bounded operators $\left\{\Phi_{t}^{\#}\right\}$ acting on $\Gamma(E)$. Let $F \subset E$ denote a continuous subbundle of $E$ which is invariant under $\Phi^{t}$. Let $\Gamma(F)$ denote the closed subspace of $\Gamma(E)$ which consists of the continuous sections of $F$. We may also form the quotient vector bundle $E / F$ and the Banach section space $\Gamma(E / F)$. Since $F$ is invariant, we can define the restriction map $L_{F}=L \mid \Gamma(F)$, where $L$ generates $\left\{\Phi_{t}^{\#}\right\}$ and $L_{F}$ is understood to act only on the intersection of the domain of $L$ and the space $\Gamma(F)$. By Proposition 1.1, the Sacker-Sell spectra have the form

$$
\Sigma=\operatorname{Re}[\sigma(L)] \text { and } \Sigma_{T}=\operatorname{Re}\left[\sigma\left(L_{F}\right)\right] .
$$


We call $\Sigma_{T}$ the tangential spectrum after the particular case in which $E=T M$, the tangent bundle, and $F=T P$, the tangent bundle of a smooth submanifold $P$ in $M$, invariant under the base flow $\phi^{t}$.

The Sacker-Sell normal spectrum is defined as follows [9]: Fix any continuous subbundle $G \subset E$ which complements $F$. Let $p_{G}$ be the projection onto $G$, then

$$
\Phi_{N}^{t}=p_{G} \Phi^{t} \mid G
$$

defines a vector bundle flow on $G$ called the normal flow. Set $\Phi_{N, \lambda}^{t}=e^{-\lambda_{t}} \Phi_{N}^{t}$, for each real number $\lambda$. Then the normal spectrum $\Sigma_{N}$ is the set of all $\lambda$ such that $\Phi_{N, \lambda}^{t}$ does not admit an exponential dichotomy over at least one $y$ in $M$. This definition is independent of the choice of complement $G$ [9]. We now match this up to the Lie spectrum.

The Lie derivative $L$ along $\Phi^{t}$ induces an operator $\hat{L}$ acting in the space $\Gamma(E / F)$ and also, in the obvious way, an operator $\bar{L}$ acting in the quotient space $\Gamma(E) / \Gamma(F)$.

Proposition 3.1. (i) $\sigma(\hat{L})=\sigma(\bar{L})$, and (ii) $\Sigma_{N}=\operatorname{Re}[\sigma(\bar{L})]$.

PRoof. The exact vector bundle sequence

$$
0 \rightarrow F \rightarrow E \rightarrow E / F
$$

determines a corresponding exact sequence of Banach spaces

$$
0 \rightarrow \Gamma(F) \rightarrow \Gamma(E) \rightarrow \Gamma(E / F) .
$$

Thus, there is a Banach space isomorphism

$$
J: \Gamma(E) / \Gamma(F) \rightarrow \Gamma(E / F),
$$

such that $J \bar{L}=\hat{L} J$. This proves (i).

Let $K$ denote the infinitesimal generator of the semigroup $\left\{\Phi_{N}^{t}\right\}^{\#}$ generated by the normal flow defined above. Then $K$ acts on sections in $\Gamma(G)$ and has the form $K \eta(x)=p_{G}(L \eta(x))$. Proposition 1.1 implies that $\Sigma_{N}=\operatorname{Re}[\sigma(K)]$. Let $\alpha: E / F \rightarrow G$ be the isomorphism determined by the splitting $E=F \oplus G$. Then $\alpha$ determines an isomorphism $A: \Gamma(E / F) \rightarrow \Gamma(G)$ such that $A \hat{L}=K A$. Therefore, $\sigma(K)=\sigma(\hat{L})$, and (ii) now follows from (i).

We come now to our main results. The next theorem is a restatement of Proposition 2.1 for the Lie derivative.

THEOREM 3.2. The Lie spectra $\sigma(L), \sigma\left(L_{F}\right)$ and $\sigma(\bar{L})$ have the following properties.

(i) $\sigma(L) \subset \sigma\left(L_{F}\right) \cup \sigma(\bar{L})$.

(ii) If $\sigma\left(L_{F}\right) \cap \sigma(\bar{L}) \subset \sigma(L)$, then

$$
\sigma(L)=\sigma\left(L_{F}\right) \cup \sigma(\bar{L}) .
$$

Corollary 3.3 [9, Theorem 1]. The spectra $\Sigma, \Sigma_{T}$ and $\Sigma_{N}$ satisfy the relation $\Sigma \subset \Sigma_{T} \cup \Sigma_{N}$.

Remark. The corollary derives from Theorem 3.2(i) and Proposition 1.2. Since the Lie spectrum need only partially intersect a given vertical line in the plane, the corollary does not seem to imply the theorem.

Since the semigroup spectrum is, up to rotation, the exponential image $e^{t \sigma(L)}$, spectral results for $\Phi_{t}^{\sharp}$ readily translate to facts about the Lie spectrum $\sigma(L)$. 
LEMMA $3.4\left[6\right.$, p. 367]. The complex number $e^{t \lambda}$ is in $\sigma_{\mathrm{ap}}\left(\Phi_{t}^{\sharp}\right)$ provided that $\sup \left\{\left|e^{-s \lambda} \Phi^{s} v\right|, s\right.$ in $\left.\mathbf{R}\right\}$ is finite for some nonzero $v$ in $E$.

Mañé [6] only establishes Lemma 3.4 for discrete tangent flows, but his methods give the lemma with minor modifications.

THEOREM 3.5. If the flow $\left\{\phi^{t}\right\}$ is chain recurrent, then

(i) $\sigma\left(\Phi_{t}^{\#}\right)=\sigma_{\mathrm{ap}}\left(\Phi_{t}^{\#}\right)$.

(ii) [9, Theorem 2] $\Sigma=\Sigma_{T} \cup \Sigma_{N}$.

Proof. For (i), it suffices to show that $\sigma\left\{\Phi_{t}^{\sharp}\right\} \subset \sigma_{\text {ap }}\left(\Phi_{t}^{\#}\right)$. If $e^{t \lambda} \in \sigma\left(\Phi_{t}^{\sharp}\right)$ then $1 \in \sigma\left(e^{-t \lambda} \Phi_{t}^{\#}\right)$ and the vector bundle flow $\left\{e^{-t \lambda} \Phi^{t}\right\}$ is not hyperbolic (see [1]). The hypothesis now implies, by [10], [11], the existence of at least one bounded bundle trajectory $\left\{e^{-s \lambda} \Phi^{s} v\right\}$ for some $v \neq 0$ in $E$. By Lemma $3.4, e^{t \lambda}$ is in $\sigma_{\text {ap }}\left(\Phi_{t}^{\sharp}\right)$. The

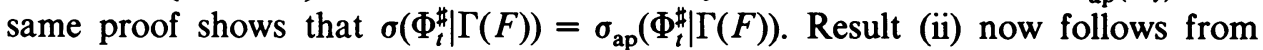
Propositions 2.1, 1.1, and 2.2.

Remarks. The condition $\Sigma=\Sigma_{T} \cup \Sigma_{N}$ may fail, as the "hull" example in [9] shows. The author has a smooth tangent flow example, based on a result of $R$. Mañé [6, Theorem A] showing that quasi-Anosov flows on submanifolds can always be extended to flows on some manifold, which are hyperbolic at the submanifold.

A result which can be derived from purely spectral hypotheses is the existence of invariant complementary subbundles. Thus, if $\Sigma_{N} \cap \Sigma_{T}=\varnothing$, then $F$ is invariantly complemented in $E$ by some subbundle $G$ [9, Theorem 3] and $\Sigma=\Sigma_{T} \cup \Sigma_{N}$. The same result follows from the operator approach by applying Proposition 2.3 to split $\Gamma(E)$ into invariant complementary subspaces. By [12], these subspaces must be given by section spaces $\Gamma(F)$ and $\Gamma(G)$ for some continuous splitting $E=F \oplus G$.

\section{REFERENCES}

1. C. Chicone and R. Swanson, The spectrum of the adjoint representation and the hyperbolicity of dynamical systems, J. Differential Equations 36 (1980), 28-40.

2. ___ Spectral theory for linearizations of dynamical systems, J. Differential Equations (to appear).

3. H. R. Dowson, Spectral theory of linear operators, Academic Press, London, 1978.

4. E. Hille and R. S. Phillips, Functional analysis and semigroups, Amer. Math. Soc. Colloq. Publ., vol. 31, Amer. Math. Soc., Providence, R. I., 1957; reprint 1974.

5. M. Hirsch, C. Pugh and M. Shub, Invariant manifolds, Lecture Notes in Math., Springer-Verlag, Berlin, 1977.

6. R. Mañé, Quasi-Anosov diffeomorphisms and hyperbolic manifolds, Trans. Amer. Math. Soc. 229 (1977), 351-370.

7. F. Riesz and B. Sz.-Nagy, Functional analysis, Ungar, New York, 1955.

8. R. Sacker and G. Sell, A spectral theory for linear differential systems, J. Differential Equations 27 (1978), 320-358.

9. __ The spectrum of an invariant submanifold, J. Differential Equations 38 (1980), 135-160.

10. _ A note on Anosov diffeomorphisms, Bull. Amer. Math. Soc. 80 (1974), 278-280.

11. J. Selgrade, Isolated invariant sets for flows on vector bundles, Trans. Amer. Math. Soc. 203 (1975), 359-390.

12. R. Swan, Vector bundles and projective modules, Trans. Amer. Math. Sooc. 105 (1962), 264-277.

Department of Mathematics, University of Missouri, Columbia, Missouri 65211 